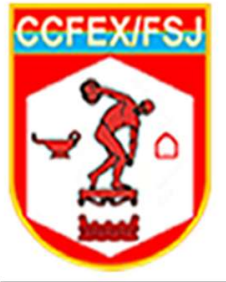

Revista de Educação Física

Joumal of Physical Education

Some page: :mnvrevistadeeduoacaofisica.0om

Artigo Original

Original Article

\title{
Aptidão física, composição corporal e autopercepção de nível de atividade física em estudantes de Educação Física: um estudo longitudinal (2015- 2018)
}

\section{Physical Fitness, Body Composition and Self-Perception of Physical Activity Level in Physical Education Undergraduates: A Longitudinal Study (2015- 2018)}

Victor Costa Valente ${ }^{1,2}$; Gustavo Costa Valente ${ }^{1}$; Mariana Passini ${ }^{1}$; Bianca Andrade Ferreira ${ }^{1}$; Marcelo Conte ${ }^{\S 1,2}$

Recebido em: 17 de dezembro de 2019. Aceito em: 17 de fevereiro de 2019

Publicado online em: 30 de abril de 2019.

\section{Resumo}

Introdução: : Na atualidade, as pessoas são incentivadas a praticar exercícios físicos e a adotar um estilo de vida ativo. Dentre os cursos da área da saúde, a Educação Física é o que apresenta o maior número de universitários com bom nível de atividade física e consequente aptidão física.

Objetivo: Comparar a aptidão física, a composição corporal e a autopercepção do nível de atividade física habitual dos alunos do curso de Educação Física da Escola Superior de Educação Física de Jundiaí (ESEF) no primeiro e no último ano de curso.

Métodos: Estudo longitudinal com uma amostra por conveniência, do qual participaram 53 alunos do curso de bacharelado em Educação Física, que foram avaliados no primeiro (2015) e no último ano (2018), por meio de anamnese, avaliações em composição corporal e em aptidão física (aptidão cardiorrespiratória, flexibilidade, resistência e força muscular). 0 teste de $t$ Student

Pontos-Chave Destaque

- Houve aumento significativo

em peso, IMC, RCQ e resistência

de membros superiores.

- O percentual de gordura

corporal diminuiu

significativamente.

- Houve uma diminuição de

$7,3 \%$ no $\mathrm{VO}_{2 m a ́ x}$ pareado foi utilizado para comparar os dados.

Resultados: Houve diferenças significativas em peso(kg), $+3,8 \%(\mathrm{p}=0,002) ; \mathrm{IMC}(\mathrm{kg} / \mathrm{m} 2)+5 \%(\mathrm{p}=0,00025)$; $\mathrm{RCQ}(\mathrm{cm})+4,9 \%(\mathrm{p}=0,00015)$; percentual de gordura corporal $(\%)-14,1 \%,(\mathrm{p}=<0,0001)$; arremesso de medicine ball $(\mathrm{cm})+6,2 \%$ ( $\mathrm{p}=0,0006)$; flexão de braços(repetições) $+10,8 \%(\mathrm{p}=0,0051) ; \mathrm{VO}_{2 \max }(\mathrm{ml} / \mathrm{kg} / \mathrm{m}),-7,3 \%$ ( $\mathrm{p}=$ 0,0384). Salto Vertical, abdominal(repetições) e flexibilidade não houve diferenças estatisticamente significativas. Conclusão: Os alunos apresentaram aumento de massa corporal, redução no percentual de gordura e melhores resultados nos testes de força e resistência muscular dos membros superiores. Entretanto, o desempenho no teste de aptidão cardiorrespiratória piorou ao longo do curso. Os resultados foram discutidos.

Palavras-chave: avaliação física, educação física, saúde.

\begin{abstract}
Introduction: Nowadays, people are encouraged to exercise and adopt an active lifestyle. Among the courses in the area of health, Physical Education is the one that presents the highest number of university students with a good level of physical activity and consequent physical fitness.
\end{abstract}

${ }^{\S}$ Autor correspondente: Victor Costa Valente - e-mail: alente_victor@hotmail.com

Afiliações: ${ }^{1}$ Escola Superior de Educação Física de Jundiaí; ${ }^{2}$ Universidade Federal de São Paulo - Departamento de Oftalmologia 
Objective: To compare the physical fitness, body composition and self-perception of the habitual physical activity level of the students of the Physical Education course of the Higher School of Physical Education of Jundiai (ESEF) in the first and last year of the course. Methods: This was a longitudinal study with a sample of convenience, in which 53 students from the baccalaureate degree in Physical Education participated, who were evaluated in the first (2015) and last year (2018), through anamnesis, body composition and physical fitness (cardiorespiratory fitness, flexibility, endurance and muscular strength). The paired Student $t$ test was used to compare the data.

Results: There were significant differences in weight $(\mathrm{kg}),+3.8 \%$ $(\mathrm{p}=0.002) ;$ BMI $(\mathrm{kg} / \mathrm{m} 2)+5 \%(\mathrm{p}=0.00025) ; \mathrm{RCQ}(\mathrm{cm})+4.9 \%(\mathrm{p}$ $=0.00015)$; percentage of body fat $(\%)-14.1 \%,(p=<0.0001)$; medicine ball pitch $(\mathrm{cm})+6.2 \%(\mathrm{p}=0.0006)$; arm flexion (repetitions) $+10.8 \%(\mathrm{p}=0.0051)$; V02 max $(\mathrm{ml} / \mathrm{kg} /$ $\mathrm{m}),-7.3 \%(\mathrm{p}=0.0384)$. Vertical jumping, abdominal (repetitions) and flexibility were not statistically significant differences.

Conclusion: The students presented increase of body mass, reduction in fat percentage and better results in tests of strength and muscular endurance of the upper limbs. However, performance in the cardiorespiratory fitness test worsened throughout the course. The results were discussed.

\section{Aptidão física, composição corporal e autopercepção de nível de atividade física em estudantes de Educação Física: um estudo longitudinal (2015- 2018)}

\section{Introdução}

Nos dias atuais, as pessoas são cada vez mais incentivadas a explorarem ao máximo a plenitude de suas capacidades físicas e a se manterem ativas. Os motivos por trás de tal fenômeno são diversos, podendo variar desde um desejo de melhorar a estética até uma necessidade de desenvolvimento funcional para a realização de atividades diárias. Especificamente, os profissionais de Educação Física são considerados importantes formadores de opinião nos aspectos relacionados ao incentivo da prática de exercícios físicos, adoção de estilo de vida saudável e manutenção da aptidão física. Nesse sentido, acredita-se que durante a respectiva formação no Ensino Superior, o estudante de Educação Física se preocupe com suas condições físicas, considerando os conhecimentos adquiridos ao longo do curso. Entretanto, observa-se que, embora estudantes universitários de cursos da área da saúde tenham um estilo de vida ativo, os alunos do curso de Educação Física são os que mais frequentemente apresentam bom nível de atividade física(1). Jesus et al.(1) mostraram que houve uma diferença estatística significativa nos níveis de atividade física entre os cursos da área da saúde, sendo que a maior parte dos estudantes de Educação Física foram classificados como fisicamente ativos e fisicamente muito ativos, enquanto entre os alunos do curso de Medicina, o sedentarismo era o estado mais prevalente. Em relação à frequência semanal de atividade física, Vargas et al.(2) revelaram que estudantes de Educação Física apresentaram melhores resultados quando comparados com estudantes de outras áreas da saúde. No que diz respeito a sexo, diversos estudos mostraram que homens matriculados no curso de Educação Física apresentaram melhores índices de atividade física do que as mulheres(2-4).

De acordo com o American College of Sports Medicine(5), as pessoas alcançam a aptidão física através do exercício físico. Ou seja, a aptidão física é um conjunto mensurável de características que são determinadas pelo 
hábito, ou falta de hábito, de um indivíduo exercitar-se. Saba(6) afirma que a resistência cardiovascular, a aptidão musculoesquelética e a composição corporal são os três componentes da aptidão física que estão relacionados à saúde. Elas podem ser adquiridas por herança genética, e desenvolvidos por meio de exercícios físicos, e também podem ser medidos e comparados. Corseuil et al.(7) realizaram um estudo que teve por objetivo identificar a prevalência de baixos níveis de aptidão física relacionada à saúde em universitários. Os resultados apontam um baixo nível de aptidão física de acordo com os testes propostos, como por exemplo, os de potência de membros inferiores, extensão de braços, flexão de tronco e força abdominal. Se tratando de indivíduos jovens, observou-se que grande parte dos universitários encontravam-se em baixos níveis de aptidão física, despertando a importância da implantação de programas para o desenvolvimento e manutenção da aptidão física. Em relação à composição corporal de estudantes universitários, um estudo com 350 acadêmicos revelou que $49,7 \%$ apresentaram resultados dentro do esperado para o Índice de Massa Corporal (IMC), 15,5\% apresentaram baixo peso e $25,4 \%$ e $9,4 \%$ apresentaram sobrepeso e obesidade, respectivamente(2).

A autopercepção sobre o nível de atividade física pode ser uma ferramenta útil para se identificar o nível de atividade física de um indivíduo. Entretanto, Barros et al.(8) relatam que estudos relacionando a autoavaliação e comportamentos relacionados à saúde ainda são escassos no Brasil.

Este estudo teve como objetivo comparar a aptidão física, a composição corporal e a autopercepção do nível de atividade física habitual dos alunos do curso de Educação Física da Escola Superior de Educação Física de Jundiaí (ESEF) no primeiro e no último ano de curso.

\section{Métodos}

\section{Desenho de estudo e amostra}

Estudo observacional, longitudinal, realizado entre os anos de 2015 e 2018, em alunos matriculados no curso de Educação Física da ESEF.
O critério de inclusão foi ter sido matriculado no primeiro semestre de 2015. Foram convidados todos os alunos recémingressos em 2015. Eram elegíveis para participar do estudo 232 alunos (142 homens e 90 mulheres). Os critérios de exclusão foram: i) recusa de participação; ii) presença de lesões no momento do teste; iii) resposta positiva ao Physical Activity Readniness Form (PAR-Q); e iv) ter sido desligado do curso.

\section{Aspectos éticos}

$\mathrm{O}$ projeto foi submetido e aprovado pelo Comitê de Ética em Pesquisa com Seres Humanos da Escola Superior de Educação Física de Jundiaí (CAAE 58771516.9.0000.5396). Todos os participantes foram esclarecidos sobre a pesquisa e o respectivo grau de envolvimento e, então, foi solicitada a assinatura do Termo de Consentimento Livre e Esclarecido, consistindo em esclarecimento a respeito dos seguintes aspectos: i) justificativa, objetivos e procedimentos utilizados; ii) desconfortos, possíveis riscos e benefícios esperados; iii) liberdade de recusar a participar ou retirar seu consentimento em qualquer fase da pesquisa, sem penalização ou prejuízo, iv) voluntariedade v) garantia de sigilo em relação aos dados coletados.

A coleta de dados se deu em dois momentos: no início do curso (2015) e ao final do curso (2018).

\section{Variáveis de estudo}

As variáveis desfecho do estudo foram: aptidão física (aptidão cardiorrespiratória, flexibilidade, resistência muscular abdominal, força / potência de membros inferiores e de membros superiores); e composição corporal.

\section{Aptidão para a prática de atividade física (anamnese)}

Para examinar a prontidão para a prática de atividade física foram aplicados dois instrumentos, antes da bateria de testes da avaliação física, nos anos de 2015 e 2018: o Physical Activity Readniness Form (PAR-Q) e o inventário de saúde quanto aos principais sinais ou sintomas sugestivos de doença cardiovascular, pulmonar ou metabólica, ambos do American College of Sports Medicine(5). 
O PAR-Q, que é um questionário sobre a condição física do avaliado, proposto para pessoas com idade entre 15 e 69 anos. Compõe-se de sete questões objetivas, com opções de resposta dicotômicas. As questões são as seguintes: 1) Seu médico já lhe disse que você é portador de uma afecção cardíaca e que somente deve realizar atividade física recomendada por um médico?; 2) Você sente dor no tórax quando realiza uma atividade física?; 3) No último mês, você teve dor torácica quando não estava realizando uma atividade física?; 4) Você perdeu o equilíbrio em virtude de uma tonteira ou já perdeu a consciência?; 5) Você sofre de algum problema ósseo ou articular (por exemplo, coluna, joelho ou quadril) que poderia ser agravado por uma mudança em sua atividade física?; 6) Seu médico está lhe receitando atualmente medicamentos (por exemplo, diuréticos) para pressão arterial ou alguma condição cardíaca?; e 7) Você está a par de alguma outra razão pela qual não deveria realizar uma atividade? As opções de resposta são sim e não para cada uma das questões. Caso a pessoa responda "sim" para uma ou mais perguntas, ela deveria procurar um médico antes do início das atividades físicas.

O inventário de saúde(5) apresenta nove possíveis sinais ou sintomas de doença e tem como objetivo estratificar os riscos do avaliado, classificando-o por categorias. Questiona-se se o indivíduo apresenta os seguintes sintomas: 1) Dor ou desconforto (tórax, pescoço, braço, maxila); 2) Falta de ar em repouso ou esforço leve; 3) Tonteira ou sincope; 4) Ortopneia ou dispneia noturna; 5) Edema nos tornozelos; 6) Taquicardia; 7) Claudicação intermitente; 8) Sopro cardíaco; 9) Fadiga incomum ou falta de ar. Os riscos são classificados em: baixo risco (quando assinalados apenas um dos sintomas), risco moderado (quando assinalados 2 ou mais sintomas) e alto risco (assinalados um ou mais sintomas e com doença cardiovascular, pulmonar ou metabólica preexistente).

Aptidão física

\section{Aptidão Cardiorrespiratória}

A captação máxima de oxigênio (VO2máx) é a medida normativa da aptidão cardiorrespiratória e, para estimá-lo, utilizou- se o protocolo do Yo-Yo Endurance Test(9), que consiste em percorrer o máximo de tempo possível, em regime de ida e volta, em um corredor com uma distância de vinte metros, em estágios progressivos e cadenciado por sinais sonoros provenientes de CD com o áudio específico do protocolo. Os valores de referência, em $\mathrm{ml} / \mathrm{kg} / \mathrm{min}$, para mulheres com idade entre 20 e 29 anos são: $<23,6$ (muito ruim), 23,6-28,9 (ruim), 29,0-32,9 (regular), 33,0-36,9 (bom), 37,0-40,9 (excelente) e $>41,0$ (superior)(10). Para os homens entre 20 e 29 anos de idade os valores, em $\mathrm{ml} / \mathrm{kg} / \mathrm{min}$, são: $<33,0$ (muito ruim), 33,0-36,4 (ruim), 36,542,4 (regular), 42,5-46,4 (bom), 46,5-52,4 (excelente) e $>52,4$ (superior)(10).

\section{Flexibilidade}

Sentar e alcançar: tem como procedimento realizar três flexões do tronco com membros inferiores estendidos até onde for possível (escala específica do banco de Wells), mantendo a posição por aproximadamente 2 segundos(11).

\section{Resistência muscular abdominal}

O teste de resistência muscular abdominal consiste em o avaliado, deitado ao solo, em decúbito dorsal, com os joelhos flexionados e membros inferiores levemente afastados, realizarão o maior número de flexões do quadril em 1 minuto, onde o mesmo encosta a região lombar no solo e tocando os cotovelos na região da coxa(11).

\section{$\underline{\text { Resistência muscular dos membros }}$}

\section{superiores}

Teste de resistência muscular dos membros superiores consiste em realizar o maior número de flexões/extensões em decúbito ventral durante trinta segundos, com os homens em quatro apoios e a mulheres em seis(11).

\section{Força / potência de membros inferiores}

$\mathrm{O}$ teste do salto vertical trata-se de medir a potência dos membros inferiores no plano vertical, cujo avaliado fica em pé com os braços estendidos para cima. O teste consiste em saltar o mais alto possível, dentro de três tentativas considerando a melhor marca. $\mathrm{O}$ resultado é dado em centímetros e o salto do avaliado é classificado em níveis de acordo com a tabela específica(12). 


\section{Força / potência de membros superiores}

Teste de arremesso de Medicine Ball tem como procedimento arremessar uma bola medicinal de três quilos. $O$ avaliado fica sentado em uma cadeira segurando a bola com as duas mãos contra o peito. $\mathrm{O}$ avaliador utiliza uma corda para segurar a cadeira e inibir a ação de embalo durante o arremesso. Diante as três tentativas, a melhor distância é computada em centímetros, e logo após o resultado é classificado(12).

\section{Composição corporal}

O procedimento para aferição do peso corporal consiste em o avaliado permanecer em pé, estando com os pés paralelos sobre a plataforma, com olhar fixo e horizontal, trajando o mínimo de vestimenta possível.

A medida da estatura foi aplicada a partir da técnica antropométrica na qual o indivíduo deve permanecer com os pés unidos, descalços, coluna ereta e olhar fixo à frente, realizando três mensurações e considerando a média.

\section{Índice de Massa Corporal (IMC)}

O Índice de Massa Corporal (IMC) é calculado a partir das medidas de peso e estatura do avaliado, cuja equação é: o peso corporal em quilogramas, dividido pela altura em metros quadrados(13). A classificação do IMC utilizada foi a preconizada pelo World Health Organization Expert Committee(13): baixo peso $(<18,5 \mathrm{~kg} / \mathrm{m} 2)$; peso adequado $(18,6 \mathrm{~kg} / \mathrm{m} 2$ a $24,9 \mathrm{~kg} / \mathrm{m} 2)$; sobrepeso (25 $\mathrm{kg} / \mathrm{m} 2$ a $29,9 \mathrm{~kg} / \mathrm{m} 2)$; obesidade $(30 \mathrm{~kg} / \mathrm{m} 2$ a $39,9 \mathrm{~kg} / \mathrm{m} 2)$ e obesidade mórbida $(>40 \mathrm{~kg} / \mathrm{m} 2)$.

\section{Relação Cintura-Quadril}

Para se estimar a relação cintura-quadril, primeiramente, é preciso medir a cintura (cicatriz umbilical) e o quadril (ponto de maior diâmetro do quadril) do avaliado. Posteriormente, a relação cintura-quadril é obtida pela divisão da circunferência da cintura pela circunferência do quadril(14).

\section{Percentual de gordura}

$O$ percentual de gordura corporal foi avaliado segundo o protocolo de Guedes(15). A mensuração é realizada em três dobras masculinas (tríceps, supra ilíaca e abdome) e três dobras femininas (subescapular, supra ilíaca e coxa).
Autoavaliação comparativa de nível de atividade física

Foi elaborado um questionário, na plataforma SurveyMonkey ${ }^{\circledR}$ para que os participantes informassem, de acordo com sua percepção, comparando o nível de atividade física no início e no final do curso, se houve aumento ou diminuição. Também foi questionado sobre o local onde eles mais se exercitaram durante esse período.

\section{Procedimentos de coleta de dados}

As coletas de dados foram realizadas exatamente no mesmo ambiente com mesma cobertura, iluminação e superfície em ambos os anos. O estudo foi apresentado em sala de aula através de slides dias antes da realização da bateria de avaliações. Nesta ocasião, diversas datas e horários foram disponibilizados para que todos tivessem a oportunidade de participar dos testes em ambos os anos. As avaliações foram compostas por uma anamnese e pelos testes de composição corporal, aptidão cardiorrespiratória, flexibilidade, resistência muscular e força explosiva.

Os avaliados foram incentivados a não realizarem exercícios físicos no mesmo dia dos testes. Os procedimentos experimentais foram realizados nas dependências da Escola Superior de Educação Física de Jundiaí. Os resultados foram analisados de acordo com cada indivíduo e o perfil físico individual elaborado.

Por ocasião da segunda coleta de dados, os alunos responderam ao questionário de autopercepção quanto ao nível de atividade física, oferecido online.

\section{Análise estatística}

Foram empregados os seguintes procedimentos estatísticos: média e desviopadrão. Em seguida, o teste de normalidade de Shapiro-Wilk e teste de homocedasticidade (critério de Bartlett). Todas as variáveis apresentaram homocedasticidade e distribuição normal e, para a comparação dos grupos, foi empregado o teste de $t$ Student pareado. Os programas computacionais utilizados foram o Excel Microsoft Windows ${ }^{\circledR}$ e o programa GraphPad®. O nível de significância adotado foi $\mathrm{p}<0,05$. 


\section{Resultados}

No ano de 2015, dos 232 alunos (142 homens e 90 mulheres) elegíveis, 221 alunos (134 homens e 87 mulheres) concordaram em participar do estudo e foram avaliados durante o primeiro semestre. Destes, apenas 53 alunos, 28 homens $(52,83 \%)$ e 25 mulheres $(47,16 \%)$, permaneceram matriculados no curso de bacharelado na ESEF e foram submetidos à mesma bateria de avaliações no ano de 2018, enquanto cursavam o último ano.

A média de idade dos participantes, ao final do curso, foi de $23,36( \pm 5,22)$ anos para os homens e $24,96( \pm 5,78)$ anos para as mulheres, e a média da estatura foi de $1,78( \pm 0,08) \mathrm{m}$ para os homens e $1,64( \pm 0,07) \mathrm{m}$ para as mulheres.

$\mathrm{O}$ peso corporal apresentou variação estatisticamente significativa exibindo um aumento de $3,82 \%(\mathrm{p}=0,05)$ para os homens e $3,84 \%(\mathrm{p}=0,01)$ para as mulheres, como se pode observar na Tabela 1 . De modo geral, todas as variáveis de composição corporal apresentaram variações com significância estatística. O IMC apresentou uma média com aumento de $4,97 \%(p=0,02)$ para os homens e $5,11 \%(p<0,01)$ para as mulheres. A relação cintura-quadril teve um aumento de $3,4 \%$ $(p=0,04)$ para os homens e $6,77 \%(p<0,01)$ para as mulheres. O percentual de gordura teve uma redução de $12,75 \%(\mathrm{p}=0,01)$ para os homens e $15,11 \%(\mathrm{p}<0,01)$ para as mulheres (Tabela 1).

Quanto à aptidão física, os resultados apresentam-se na Tabela 2. Quanto à aptidão cardiorrespiratória, as médias alcançadas em $2015(29,48 \mathrm{ml} / \mathrm{kg} / \mathrm{min})$ e em 2018 $(25,76 \mathrm{ml} / \mathrm{kg} / \mathrm{min})$ nas mulheres; de acordo com a faixa etária, foram classificadas como regular e ruim, respectivamente. Os homens apresentaram um valor médio de 38,76 $\mathrm{ml} / \mathrm{kg} / \mathrm{min}$ em 2015 e $37,37 \mathrm{ml} / \mathrm{kg} / \mathrm{min}$ em 2018, enquadrando-se na classificação de regular.

Em ambos os sexos, não houve diferença estatisticamente significativa $(p>0,05) \quad \mathrm{em}$ : força/potência de membros inferiores; resistência abdominal, flexibilidade e aptidão cardiorrespiratória. Houve ganho de força/resistência de membros superiores somente no sexo feminino com um aumento de $18,24 \%(p=0,01)$. Força/potência de membros superiores aumentou somente entre os homens, com um aumento registrado de $8,37 \%$ $(\mathrm{p}<0,01)$.

Conforme demonstrado na Tabela 2, os resultados no teste de aptidão cardiorrespiratória diminuíram em 3,57\% ( $\mathrm{p}=$ $0,29)$ para os homens e $12,61 \%(\mathrm{p}=0,08)$ para as mulheres.

O questionário online mostrou que $46,42 \%$ dos homens e $48,00 \%$ das mulheres responderam que seus níveis de atividade física aumentaram após estarem matriculados no curso de Educação Física (Tabela 3). Na questão sobre o local em que eles mais se exercitaram durante o período do curso, $71,42 \%$ dos homens e $72,00 \%$ das mulheres selecionaram a resposta Academia (Tabela 4).

\section{Discussão}

Ao decorrer dos anos de curso, os alunos apresentaram aumento de massa corporal, porém uma diminuição no percentual de gordura. Estes resultados indicam que houve um aumento de massa magra, mais possivelmente da massa muscular. Fato que pode ser evidenciado pela melhora nos resultados nos testes de força/potência muscular dos membros superiores para os homens e força/resistência muscular dos membros superiores para as mulheres. Entretanto, os mesmos alunos apresentaram piores desempenhos nos testes de aptidão cardiorrespiratória. Conte et al.(16), em um estudo com 290 universitários do curso de Medicina, utilizaram o teste de pista, composto por corrida em 2.400 metros, para avaliarem a aptidão cardiorrespiratória dos estudantes. Os resultados foram de $25,98 \mathrm{ml} / \mathrm{kg} / \mathrm{min}$ para as mulheres e $35,87 \mathrm{ml} / \mathrm{kg} / \mathrm{min}$ para os homens, revelando valores de VO2máx semelhantes ao do presente estudo. De acordo com o referencial proposto para essa idade, as mulheres deveriam apresentar valores $>37,0$ $\mathrm{ml} / \mathrm{kg} / \mathrm{min}$ para serem consideradas excelentes e os homes valores acima de 46,5 $\mathrm{ml} / \mathrm{kg} / \mathrm{min}(10)$. Resultados classificados regulares e ruins nos testes de $\mathrm{VO}_{2 \text { máx }}$ podem ser preocupantes, pois, segundo Ribeiro(17), indivíduos que apresentam baixa capacidade cardiorrespiratória podem desenvolver diver - 
Tabela 1 - Características descritivas e composição corporal da amostra - resultados da comparação dos dados dos alunos de Educação Física nos anos de 2015 e $2018(\mathrm{~N}=53)$

\begin{tabular}{|c|c|c|c|c|c|c|c|c|c|c|}
\hline \multirow[t]{2}{*}{ Característica } & \multicolumn{5}{|c|}{ Homens } & \multicolumn{5}{|c|}{ Mulheres } \\
\hline & Média 2015 & DP & Média 2018 & DP & $\boldsymbol{P}$ & Média 2015 & DP & Média 2018 & DP & $\boldsymbol{P}$ \\
\hline Idade, anos & 20,25 & $\overline{5,23}$ & 23,36 & 5,22 & - & 21,68 & 5,94 & 24,96 & 5,78 & - \\
\hline Peso, quilogramas & 74,11 & 11,57 & 76,94 & 12,94 & 0,05 & 60,12 & 6,59 & 62,43 & 7,43 & $\mathbf{0 , 0 1}$ \\
\hline Estatura, metros & 1,77 & 0,07 & 1,78 & 0,08 & $\mathbf{0 , 3 3}$ & 1,63 & 0,06 & 1,64 & 0,07 & $\mathbf{0 , 8 4}$ \\
\hline $\mathrm{IMC}^{\mathrm{a}}$ & 23,67 & 2,94 & 24,84 & 3,48 & 0,02 & 22,45 & 1,91 & 23,60 & 2,67 & $<0,01$ \\
\hline $\mathrm{RCQ}^{\mathrm{b}}$, centímetros & 0,83 & 0,05 & 0,86 & 0,06 & 0,04 & 0,75 & 0,05 & 0,80 & 0,06 & $<0,01$ \\
\hline Gordura corporal, \% & 17,97 & 5,00 & 15,68 & 6,28 & $\mathbf{0 , 0 1}$ & 28,60 & 2,99 & 24,28 & 3,52 & $<0,01$ \\
\hline
\end{tabular}

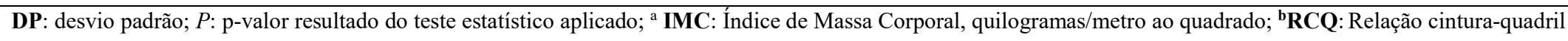

Tabela 2 - Aptidão física em alunos de Educação Física ao ingresso no início (2015) e ao término do curso (2018) (N=53)

\begin{tabular}{|c|c|c|c|c|c|c|c|c|c|c|}
\hline \multirow[t]{2}{*}{ Valência Física } & \multicolumn{5}{|c|}{ Homens } & \multicolumn{5}{|c|}{ Mulheres } \\
\hline & Média 2015 & DP & Média 2018 & DP & $\boldsymbol{P}$ & Média 2015 & DP & Média 2018 & DP & $P$ \\
\hline $\begin{array}{c}\text { Força/potência de membros } \\
\text { inferiores }^{\mathrm{a}}\end{array}$ & 49,57 & 15,35 & 45,39 & 8,37 & $\mathbf{0 , 1 3}$ & 34,60 & 4,96 & 34,28 & 6,69 & $\mathbf{0 , 8 3}$ \\
\hline $\begin{array}{c}\text { Força/potência de membros } \\
\text { superiores }^{\mathrm{b}}\end{array}$ & 473,43 & 66,28 & 513,04 & 68,89 & $<0,01$ & 307,20 & 50,00 & 314,56 & 40,05 & 0,49 \\
\hline Resistência muscular abdominal $^{\mathrm{c}}$ & 42,21 & 9,34 & 39,52 & 11,43 & $\mathbf{0 , 2 1}$ & 31,96 & 5,73 & 35,08 & 8,07 & $\mathbf{0 , 0 8}$ \\
\hline Flexão de braços, repetições & 25,61 & 7,59 & 27,32 & 6,92 & $\mathbf{0 , 1 7}$ & 18,17 & 4,37 & 21,48 & 5,14 & $\mathbf{0 , 0 1}$ \\
\hline Flexibilidade, centímetros & 30,14 & 7,88 & 30,00 & 6,66 & 0,91 & 33,40 & 6,27 & 32,08 & 7,27 & 0,27 \\
\hline Aptidão cardiorrespiratória $^{\mathrm{d}}$ & 38,76 & 7,57 & 37,37 & 8,41 & $\mathbf{0 , 2 9}$ & 29,48 & 6,15 & 25,76 & 7,44 & 0,08 \\
\hline
\end{tabular}

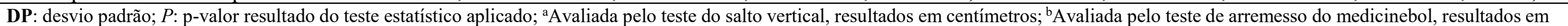

centímetros; ${ }^{c}$ Teste abdominal, resultado em número de repetições; ${ }^{d}$ Resultados em VO2máx em mililitros/quilograma/minuto, avaliado pelo Yo yo test. 
Tabela 3 - Autopercepção do nível de atividade física dos alunos após o ingresso no ensino superior

\begin{tabular}{lcccc}
\hline \multirow{2}{*}{ Descrição do Item } & \multicolumn{2}{c}{ Homens } & \multicolumn{2}{c}{ Mulheres } \\
\cline { 2 - 5 } & Frequência & \% & Frequência & \% \\
\hline \hline Aumentou & 13 & 46,42 & 12 & 48,00 \\
Diminuiu & 6 & 21,42 & 5 & 20,00 \\
Permaneceu igual & 9 & 32,14 & 8 & 32,00 \\
\hline
\end{tabular}

Tabela 4 - Questionário sobre o local em que os alunos mais se exercitaram durante o período do

\begin{tabular}{lcccc}
\hline \multirow{2}{*}{ Descrição do Item } & \multicolumn{2}{c}{ Homens } & \multicolumn{2}{c}{ Mulheres } \\
\cline { 2 - 5 } & Frequência & \% & Frequência & \% \\
\hline \hline Clube & 2 & 7,14 & 1 & 4,00 \\
Academia & 20 & 71,42 & 18 & 72,00 \\
Faculdade & - & - & 1 & 4,00 \\
Rua & 2 & 7,14 & 1 & 4,00 \\
Outros & 4 & 14,28 & 4 & 16,00 \\
\hline
\end{tabular}

sos riscos à saúde, incluindo incapacidade física e doenças cardiovasculares e respiratórias.

De acordo com Haskell(18), a realização de atividades físicas com intensidade e volume acima do mínimo recomendado pode causar efeitos benéficos para a saúde e aptidão física do indivíduo. Os exercícios, realizados majoritariamente nas academias segundo a Tabela 4, podem ter proporcionado um aumento na massa muscular dos alunos, consequentemente os mesmos obtiveram uma melhor performance nos testes de força e resistência dos membros superiores. Diferente do presente estudo, Corseuil et al.(7) observaram, em um estudo com 234 universitários, que as maiores prevalências de baixa aptidão foram encontradas nos testes da força muscular em ambos os sexos.

$\mathrm{Na}$ comparação com os resultados preconizados pelas tabelas de Percentual de Gordura, segundo sexo e idade, o percentual de gordura dos alunos avaliados mostra resultados médios em ambos os sexos e anos(5). O porcentual de gordura corporal é uma importante medida, pois segundo Tritschler(19), pessoas com excesso de gordura corporal tem maior probabilidade de apresentar doenças cardiovasculares e outros tipos de doenças graves. De acordo Moreira et al.(20), o sobrepeso e a obesidade são fatores de risco que podem ser atenuados através do treinamento de resistência aeróbica.

Em relação ao IMC, os alunos apresentaram resultados classificados como adequados em ambos os sexos e idades(13). Similares aos resultados do presente estudo, Vargas et al.(2), em amostra com 350 universitários de distintos cursos, revelaram que metade dos participantes estudados apresentaram resultados dentro do esperado para o IMC.

Outra valência importante na aptidão física dos universitários avaliados é a flexibilidade. De acordo com Riestre et al.(21), bons resultados em flexibilidade são importantes para um melhor desempenho em outras valências como força, resistência e potência. Por isso, a flexibilidade deve ser levada em consideração pelos profissionais de Educação Física na prescrição de exercícios físicos.

Quanto a autopercepção do nível de atividade física, grande parte dos estudantes de Educação Física, em ambos os sexos, afirmaram que acreditam que seus níveis de atividade física aumentaram após se tornarem universitários. Em contrapartida, um estudo com 194 estudantes universitários de Educação Física revelou que o nível de atividade física mantém-se constante durante o período do curso(4). 
Segundo Iriart et al.(22), nos tempos atuais, o corpo humano tornou-se um objeto de consumo. Esse processo, influenciado pela mídia que tem definido valores e padrões, tem alimentado a milionária indústria da estética e levado inúmeros indivíduos a utilização de anabolizantes para um ganho de massa muscular. Sendo assim, após a análise dos resultados do presente estudo, pode-se sugerir que o culto ao corpo e a busca pela melhora puramente estética tem impactado negativamente a condição cardiorrespiratória dos alunos. Ou seja, os alunos, preocupados somente com a estética, tem diminuído sua preocupação ou menosprezado a saúde cardiorrespiratória, visto que o aumento da massa muscular, a diminuição do percentual de gordura e a piora nos níveis VO2máx ficaram evidentes no estudo. Esses resultados são muito relevantes, pois levam a reflexão do papel do profissional de Educação Física, incluindo os professores universitários da área, na conscientização de seus alunos sobre a importância da prática de exercícios que gerem um impacto positivo na condição cardiorrespiratória para a manutenção da saúde dos mesmos.

Na sociedade contemporânea, ouve-se falar com frequência sobre a importância da prática de exercícios físicos para a melhora da saúde, devido a resultados de estudos que mostram que o exercício físico previne de maneira eficaz a mortalidade prematura(14). Entretanto, é necessário levar em consideração alguns detalhes importantes antes do início das atividades. Segundo Pitanga(23), saúde não é apenas a ausência de doenças, mas também uma multiplicidade de aspectos do comportamento humano voltados a um estado de completo bem-estar físico, mental e social. Por esse motivo, é essencial que todo e qualquer exercício seja prescrito por um profissional de Educação Física que esteja habilitado para identificar o potencial e as limitações de cada indivíduo. Consequentemente, o esporte e/ou o exercício físico devem ser praticados de forma periodizada a fim de equilibrar o volume e a intensidade dos treinos, evitando assim exageros que podem levar ao abandono da atividade ou a nenhuma adaptação fisiológica devido à falta de intensidade. Além disso, excesso de treinos e pouco descanso podem sobrecarregar o indivíduo fisicamente $\mathrm{e}$ psicologicamente, muitas vezes, ocasionando problemas como o overtraining (excesso de treinamento).

Por essa razão, novas estratégias podem ser criadas nas instituições de ensino superior para que os alunos tenham a oportunidade de melhorar suas valências físicas durante os anos de curso, já que apenas $2,5 \%$ dos participantes no estudo disseram que a faculdade foi o local em que eles mais se exercitaram durante o período de formação. Jesus et al.(1), sugerem que exista dentro das instituições de ensino superior mais incentivo a prática de atividades física e a disseminação de informações que levem os estudantes a adotarem um estilo de vida mais saudável. Os professores da ESEF também podem conscientizar os alunos sobre a importância de manter uma boa condição cardiorrespiratória para a manutenção da saúde já que os mesmos obtiveram resultados de $\mathrm{VO}_{2 \text { máx }}$ abaixo do esperado em ambos os sexos, o que pode ser preocupante visto que a relação entre níveis baixos de aptidão cardiorrespiratória e doenças cardiovasculares fica evidente na literatura que trata do assunto.

\section{Pontos fortes e limitações do estudo}

Os alunos que participaram do estudo como voluntários e avaliados puderam experimentar algo relevante a sua formação, pois se envolveram em situações reais da área, com possibilidade de vivenciar os procedimentos típicos da Educação Física, bem como analisar os dados das respectivas avaliações físicas. $\mathrm{O}$ presente estudo utilizou protocolos de avaliação física que possuem tabelas referenciais representando populações específicas, muitas vezes, com características diferentes da população avaliada. Assim sendo, sugere-se a implementação de ações educativas para orientação desses alunos a respeito da importância da manutenção da aptidão física, bem como a realização de outras investigações no mesmo âmbito.

\section{Conclusão}

O presente estudo examinou a evolução da aptidão física de alunos no momento do ingresso até o final do curso de bacharelado em Educação Física. No decorrer dos anos de 
curso, os alunos apresentaram aumento de massa corporal, porém houve redução no percentual de gordura e melhores resultados nos testes de força e resistência muscular dos membros superiores. Entretanto, o desempenho no teste de aptidão cardiorrespiratória piorou ao longo do curso.

A avaliação das variáveis antropométricas e aptidão física dos alunos permitiu estabelecer o perfil do grupo para sugerirem-se intervenções. Os ingressantes ao curso de Educação Física apresentaram resultados médios dentro da maioria das valências físicas avaliadas, entretanto obtiveram resultados de VO2max abaixo do esperado em ambos os sexos.

Os resultados da avaliação das variáveis antropométricas e aptidão física dos alunos apresentados podem ser relevantes para a sociedade e para os professores da ESEF, pois é possível analisar a influência que suas aulas, juntamente com outros fatores externos, têm sobre a aptidão física de seus alunos.

Por fim, poderão ser criados não somente um núcleo acadêmico-científico na instituição, atingindo o público de interesse para pesquisa, mas também parcerias com grupos ligados a saúde. Desta maneira, um pensamento crítico é criado para reconhecer a relevância da avaliação física, gerando um ganho na formação profissional.

\section{Agradecimentos}

Os autores agradecem a todos os voluntários do estudo, não somente os avaliadores, mas também a todos os alunos que participaram das baterias de avaliações. Além disso, um agradecimento especial deve ser destinado a ESEF por ter cedido suas instalações para a realização da pesquisa e por ter facilitado a realização da mesma juntamente com seu corpo docente.

\section{Agradecimentos}

Os autores agradecem a todos os voluntários do estudo, não somente os avaliadores, mas também a todos os alunos que participaram das baterias de avaliações. Além disso, um agradecimento especial deve ser destinado a ESEF por ter cedido suas instalações para a realização da pesquisa e por ter facilitado a realização da mesma juntamente com seu corpo docente.

\section{Declaração de conflito de interesses}

Não existe nenhum conflito de interesses no presente estudo.

\section{Declaração de financiamento}

A pesquisa teve o financiamento do Conselho Nacional de Desenvolvimento Científico e Tecnológico (CNPq).

\section{Referências}

1. Jesus CF, Oliveira RAR, Badaró AC, Ferreira EF. Nível de atividade física de estudantes da área da saúde de uma instituição superior particular de UBÁ-MG. Revista Brasileira de Prescrição e Fisiologia do Exercício. [Online] 2017; 68:565-573. Disponível em: http://www.rbpfex.com.br/index.php/rbpfe $\mathrm{x} /$ article/view/1198. [Acessado em: 10/11/2018].

2. Vargas LM, Cantorani JRH, Vargas TM, Gutierrez GL. Fatores associados ao nível de atividade física entre estudantes universitários. Revista Brasileira de Qualidade de Vida. [Online] 2015; 3:158168. Disponível em: doi: 10.3895/rbqv.v7n3.3217.

3. Rodrigues HWG, Júnior MB, Wilke N. Nível de atividade física em estudantes do curso de Educação Física: um estudo de caso. EFDeportes.com, Revista Digital. [Online] 2010; 15:148. Disponível em: http://www.efdeportes.com/efd148/ativida de-fisica-em-estudantes-de-educacaofisica.htm. [Acessado em: 10/11/2018].

4. Silva GSF, Bergamaschine R, Rosa M, Melo C, Miranda R, Filho MB. Avaliação do nível de atividade física de estudantes de graduação das áreas saúde/biológica. Revista Brasileira de Medicina do Esporte. [Online] 2007; 13:1. Disponível em: doi: $10.1590 / \mathrm{S} 1517-86922007000100009$.

5. American College of Sports Medicine. Diretrizes do ACSM para os Testes de Esforço e sua prescrição. $8^{\mathrm{a}}$ ed. Rio de Janeiro: Guanabara Koogan; 2010. p. 12, $14,19,43$.

6. Saba F. Mexa-se: atividade fisica, saúde e bem estar. $1^{a}$ edição. São Paulo: Takano Editora; 2003. 
7. Corseuil W, Petroski EL. Baixos níveis de aptidão física relacionada à saúde em universitários. Revista Brasileira de Educação Física e Esporte. [Online] 2010; 24(1): 49-54. Disponível em: doi: 10.1590/S1807-55092010000100005.

8. Barros MB, Zanchetta LM, de Moura EC, Malta DC. Auto-avaliação da saúde e fatores associados, Brasil, 2006. Revista de Saúde Pública. [Online] 2009; 43: 27-37. Disponível em: doi: 10.1590/S003489102009000900005.

9. Krustrup P, Mohr M , Amstrup T, Rysgaard $\mathrm{T}$, Johansen J , Steensberg A, et al. The yoyo intermittent recovery test: physiological response, reliability, and validity. Medicine and Science in Sports Exercise. [Online] 2003; 35:697-705. Disponível em: doi: 10.1249/01.MSS.0000058441.94520.32.

10. Powers SK, Howley ET. Fisiologia do exercício: teoria e aplicação ao condicionamento e ao desempenho. São Paulo: Manole; 2000. p. 270.

11. Mathews DK. Medida e avaliação em Educação Física. Rio de Janeiro: Guanabara Koogan; 1986. p. 328, 113, 126.

12. Marins JCB, Giannichi RS. Avaliação e prescrição de atividade física: guia prático. $2^{\mathrm{a}}$ ed. Rio de Janeiro: Shape; 1998. p. 87, 91.

13. World Health Organization Expert Committee. Physical Status: the use and interpretation of anthropometry. [Online] 1995; Technical Report Series number 854. Disponível em: http://www.who.int/childgrowth/publicatio ns/physical_status/en/ [Acessado em: 09/04/2018].

14. McArdle WD, Katch FI, Katch VL. Fisiologia do exercício: energia, nutrição e desempenho humano. Rio de Janeiro: Guanabara; 2003. p. 796, 913.

15. Guedes DP. Estudo da gordura corporal através da mensuração dos valores de densidade corporal e da espessura de dobras cutâneas em universitários. Revista Kinesis. [Online] 1985; 1(2): 183-212. Disponível em: doi: $10.5902 / 23165464$.
16. Conte M, Gonçalves A, Chalita LVAS, Ramalho LCB. Nível de Atividade Física como Estimador da Aptidão Física de Estudantes Universitários: Explorando a Adoção de Questionário Através de Modelagem Linear. Revista Brasileira de Medicina do Esporte. [Online] 2008; vol. 14, n. 4. Disponível em: http://www.scielo.br/pdf/rbme/v14n4/v14n 4a02.pdf. [Acessado em 12/11/2018].

17. Ribeiro FV. Educação Física, seu manual de saúde. São Paulo: Difusão Cultura do Livro; 2012. p.77.

18. Haskell WL, Lee I, Pate RR, Powell KE, Blair SN, Franklin BA, et al. Physical activity and public health: Updated recommendation for adults from the American College of Sports Medicine and the American Heart Association. Medicine and Science in Sports Exercise. [Online] 2007; 39(9): 1.423-1.434. Disponível em: doi: 10.1249/mss.0b013e3180616b27.

19. Tritschler K. Medida e avaliação em Educação Física e esportes de Barrow \& McGee. $5^{\mathrm{a}}$ ed. Barueri: Manole; 2003. p. 229.

20. Moreira WW, Simões R. Esporte como fator de qualidade de vida. Piracicaba: Unimep; 2002. p.68.

21. Riestre A, FLIX J. 1.004 Exercícios de flexibilidade. $5^{\text {a }}$ ed. Porto Alegre: ArtMed; 2003. p.29.

22. Iriart JAB, Chaves JC, Orleans RG. Culto ao corpo e uso de anabolizantes entre praticantes de musculação. Cadernos de Saúde Pública. [Online] 2009; 25(4): 773782. Disponível em: doi: 10.1590/S0102311X2009000400008.

23. Pitanga FJG. Epidemiologia, atividade física e saúde. Revista Brasileira de Ciências e Movimento. [Online] 2002; 10(3): 49-54. Disponível em: https://portalrevistas.ucb.br/index.php/RB CM/article/viewFile/463/489. [Acessado em: 12/04/2018]. 